\title{
LncRNA HOTAIR Increases Inflammatory Responses by Activating NF-KB Pathway in LPS- Induced Acute Lung Injury
}

\section{XiaoMei Huang}

Guangzhou First People's Hospital

\section{ZeXun Mo}

Guangzhou First People's Hospital

\section{YuJun Li}

Guangzhou First People's Hospital

Hua He

Guangzhou First People's Hospital

\section{KangWei Wang}

Guangzhou First People's Hospital

\section{NingNing $\mathrm{Xu}$}

Guangzhou First People's Hospital

\section{WeiHong Guo}

Guangzhou First People's Hospital

\section{WanNa Tang}

Guangzhou First People's Hospital

Shuquan Wei ( $\nabla$ eyweishuquan@scut.edu.cn)

Guangzhou First People's Hospital https://orcid.org/0000-0003-4044-2244

\section{Research article}

Keywords: Acute lung injury, Inflammatory, HOX transcript antisense RNA, Nuclear factor kappa-B

Posted Date: August 27th, 2020

DOI: https://doi.org/10.21203/rs.3.rs-49969/v1

License: (c) (1) This work is licensed under a Creative Commons Attribution 4.0 International License. Read Full License 


\section{Abstract}

Background Nuclear factor kappa-B (NF-kB) activation increased the expression of cytokines and further lead to lung injury was considered the main mechanism of acute lung injury (ALI). Here, we focus on exploring the potential regulatory mechanism between long noncoding RNA (LncRNA) HOX transcript antisense RNA (HOTAIR) and NF-KB on LPS-induced ALI.

Methods A549 cells were then divided into 4 groups: HOTAIR group, NC group, si-HOTAIR group and si-NC group. These 4 groups were then treated with $1 \mu \mathrm{g} / \mathrm{mL}$ lipopolysaccharides (LPS) or without LPS at $37^{\circ} \mathrm{C}$ for $24 \mathrm{~h}$. The expression level of cytokines (tumor necrosis factor (TNF)-a, interleukin (IL)-1 $\beta$ and IL-6) and LncRNA HOTAIR were evaluated by quantitative Real Time Polymerase Chain Reaction (qRT-PCR) and Enzyme-linked immunosorbent assay (ELISA). Western Blot analysis was adopted for evaluating the level of $\mathrm{p}-\mathrm{I} \mathrm{KBa} / \mathrm{IkBa}$ and $\mathrm{p}-\mathrm{p} 65 / \mathrm{p} 65$. Nuclear translocation of p65 was observed by immunofluorescence staining.

Results qRT-PCR and ELISA assay showed that the expression of cytokines (IL-1 $\beta$, IL- 6 and TNF- $\alpha$ ) and inflammatory gene HOTAIR was remarkably increased with LPS treatment $(p<0.01)$. Over-expression of HOTAIR significantly increased the expression of cytokines (including IL-1 $\beta$, IL- 6 and TNF- $a$ ) and NF-KB pathway associated proteins (including $p-\mathrm{IKBa}_{\mathrm{K}} \mathrm{IkBa}$ and $\mathrm{p}-\mathrm{p} 65 / \mathrm{p} 65$ ), while knockdown of HOTAIR had the opposite effect $(p<0.01)$. The immunofluorescence assay showed that the level of $p 65$ in the nucleus was significantly higher in the HOTAIR group and significantly lowers in the si-HOTAIR group $(p<0.01)$.

Conclusion HOTAIR may play a pro-inflammatory response through NF-KB pathway in LPS-induced ALI, which may provide a perspective for further understanding the pathogenic mechanism of ALI.

\section{Background}

ALI is a morbid pulmonary inflammatory disease which may lead to acute respiratory failure diseases [1]. Bacterial infection was considered the main cause of ALI, in which LPS on bacteria could activate NF-KB signaling pathway through TLR4 signals and further lead to the enhancement of cytokines expression and further lead to lung injury [2] [3] [4]. Thus, restrain inflammatory reaction via TLR4 signaling was suggested a possible mechanism of ALI treatment [5].

LncRNAs were able to regulate varied genes expressed in cells and tissues [6]. It is reported that multiple IncRNAs have contribute to the progress of ALI, for example, high levels of the IncRNAs nuclear paraspeckle assembly transcript 1 (NEAT1) aggravated the progression of ALI via HMGB1/RAGE signaling, increased metastasis-associated lung adenocarcinoma transcript 1 (MALAT1) was positively correlated with increased severity and unfavorable prognoses via TSC2-mTOR and NF-KB signaling pathways[7][8][9][10]. Long noncoding RNA taurine up-regulated gene 1 (TUG1) targeted to miR-34b-5p and activated GRB2 associated binding protein 1 (GAB1), which showed a reducing pulmonary injury in ALI mice [11]. However, specific prognostic and therapeutic target of IncRNAs in ALI remain uncertain. 
LncRNA HOTAIR have impacts on multiple cancer diseases [12]. It was regarded as a potential prognostic and therapeutic target in lung cancer [13]. Until recently, the role of HOTAIR in LPS-induced ALI remains unknown. Here, we presented a research focus on exploring the potential regulatory mechanism between HOTAIR and NF-KB signaling pathway on LPS-induced ALI.

\section{Methods}

\subsection{Cell culture and LPS treatment}

EML4-ALK Fusion-A549 Isogenic Cell Line Human (ATCC ${ }^{\circledR}$ CCL-185IG ${ }^{\mathrm{TM}}$ ) was purchased from American Type Culture Collection (ATCC) (ATCC, Manassas, VA, USA). Cells were cultured in complete medium of DMEM with $10 \%$ FBS and $1 \%$ penicillin streptomycin at $37^{\circ} \mathrm{C}$ under $5 \%$ CO2. LPS induced ALI cell model was induced by incubated with $1 \mu \mathrm{g} / \mathrm{mL}$ LPS (Escherichia coli 055:B5, Sigma Aldrich, Saint Louis, Missouri, USA) at $37^{\circ} \mathrm{C}$ for $24 \mathrm{~h}$ as described in our previous report [14].

\subsection{Cell transfection}

pcDNA 3.1-HOTAIR (HOTAIR group), pcDNA 3.1 empty vector (NC group), siRNAs specifically targeting HOTAIR (si-HOTAIR) and siRNA negative control (si-NC) were purchased from Sigma. MH-S and MLE-12 cells were seeded into 96 -well plates and cultured for overnight at $37^{\circ} \mathrm{C}$. All of these oligonucleotides were transfected into A549 cells using Lipofectamine 2000 reagent (Invitrogen, Carlsbad, CA, USA) incubated for $48 \mathrm{~h}$ according to the manufacturer's protocols.

\section{3 qRT-PCR analysis}

Total RNAs were isolated using TRIzol reagent (Invitrogen, Carlsbad, CA, USA). The RNAs were used for synthesizing cDNA with a miRcute miRNA first-strand cDNA synthesis kit (TIANGEN Biotech, Beijing, China) and eliminating gDNA with PrimeScript RT Reagent Kit with gDNA Eraser (TIANGEN Biotech, Beijing, China). IL-1 $\beta$, IL-6, TNF- $\alpha$ and LncRNA HOTAIR were evaluated using Power SYBR ${ }^{\circ}$ Green PCR Master Mix (Applied Biosystems, Foster City, CA, USA) with custom designed primers (Table 1). The relative expression was normalized with glyceraldehyde-3-phosphate dehydrogenase (GAPDH) qRT-PCR was performed on a 7500 Real-Time PCR system (Applied Biosystems, USA) and conducted under the following conditions: $95^{\circ} \mathrm{C}$ for $5 \mathrm{~min}, 40$ cycles of $95^{\circ} \mathrm{C}$ for $15 \mathrm{~s}$ and $60^{\circ} \mathrm{C}$ for $60 \mathrm{~s}$. The experiment was performed in triplicate. The relative gene expression was calculated $d$ using the $2^{-\triangle \Delta C T}$ method and normalized to the expression of internal control. 
Table 1

primers

\begin{tabular}{|llll|}
\cline { 2 - 4 } & \multicolumn{2}{c|}{ Forward } & Reverse \\
HOTAIR & CAGTGGGGAACTCTGACTCG & GTGCCTGGTGCTCTCTTACC \\
IL-1 $\beta$ & CTTGGTGATGTCTGGTCCAT' & CCTTGTACAAAGGACATGGAG \\
IL-6 & CCAGAGCTGTGCAGATGAGT & CTGCAGCTTCGTCAGCAGGC \\
TNF-a & CCCACCTTTGCCCGGGGTTC' & CCTCCCGGGCGTCAGCACTA \\
GAPDH & GCTCATTTGCAGGGGGGAG & GTTGGTGGTGCAGGAGGCA- \\
\hline
\end{tabular}

\subsection{ELISA analysis}

Proteins ofA549 cells was isolated with lysis buffer (Elabscience, Wuhan, China) and the concentration was confirmed with Pierce ${ }^{\mathrm{TM}}$ BCA protein assay kit (Elabscience, Wuhan, China). The level of IL-1 $\beta$, IL-6 and TNF-a were detected using ELISA kits (Beyotime Biotechnology, Shanghai, China) on a multiskan FC spectrum system (Thermofish, USA) and measured at an absorbance at $450 \mathrm{~nm}$.

\subsection{Western Blot Assay}

Total cellular proteins were isolated with pre-cold RIPA buffer (Beyotime Institute of Biotechnology, Nantong, China) with protease inhibitor. The proteins were separated by SDS-PAGE electrophoresis and transferred into polyvinylidene fluoride (PVDF) membranes and fixed in PBS with $0.1 \%$ Tween-20 (PBST) containing $5 \%$ non-fat milk for $2 \mathrm{~h}$ at room temperature. After the block, the membrane was incubated with primary antibodies (anti-p-IKBa monoclonal antibody (1:1,000; abcam, No. ab133462), anti-IKBa monoclonal antibody (1:1,000; abcam, No. ab32518), p-p65 NF-kB monoclonal antibody (1:10,000; abcam, No. ab76302), anti-p65 NF-kB monoclonal antibody(1:10,000; abcam, No. ab732536), and antihuman GAPDH polyclonal antibody (1:10,000; abcam, No. ab181602,)at $4^{\circ}$ Covernight and then incubated with the horseradish peroxidase (HRP) labeled secondary antibody $(1: 10,000)$ at $25^{\circ} \mathrm{C}$ for $2 \mathrm{~h}$. GAPDH was used as the loading control. The protein bands were detected using the enhanced chemiluminescence reagent (Thermo Scientific, USA).

\subsection{Immunofluorescence analysis}

A549 cells were seeded on a microscope slide and fixed in 4\% paraformaldehyde (PFA) for $15 \mathrm{~min}$ at room temperature. Then we blocked the cells with $1 \times$ PBS containing $5 \%$ goat normal serum and $0.3 \%$ Triton- $\times 100$ for $1 \mathrm{~h}$. The cells were then incubated with rabbit anti-p65 NF-kB primary antibodies (1:200, 710048, Ebioscience, US) at $4{ }^{\circ} \mathrm{C}$ overnight and Anti-Goat secondary antibody (Ebioscience, US) for $1 \mathrm{~h}$ at room temperature. Finally, the cells were stained with DAPI for $10 \mathrm{~min}$ and mounted with fluorescent mounting solution. Images were taken by fluorescence microscope with excitation wavelength of $360 \mathrm{~nm}$ (Nikon ECLIPSE TE2000-U).

\subsection{Statistical analysis}


Continuous variables are presented as mean \pm standard deviation (SD). Difference between control and LPS-induced group was performed through student's $t$ test and the differences between multiple groups were analyzed using a one-way analysis of variance (ANOVA) followed by a post-hoc LSD test. Statistical analyses were performed with the statistical package for the social sciences (SPSS) version 22.0 software (IBM Inc., USA) and P-values $<0.05$ were considered statistically significant.

\section{Results}

\section{LPS induced increasing IL-1 $\beta$, IL-6, TNF- $\alpha$ and IncRNA HOTAIR}

A LPS-induced ALI cell model was established and the expression of cytokines (IL-1 $\beta$, IL-6, TNF- $a$ ) and inflammatory gene HOTAIR were detected by both qRT-PCR and ELISA, which have been described as our previous report [14]. The results showed that the expression of IL-1 $\beta$, IL-6 and TNF- $a$ (Fig. 1a \& Fig. 1b) and HOTAIR (Fig. 1C) was remarkably increased with LPS treatment compared with the control group.

\section{LncRNA HOTAIR correlated with inflammation response induced by LPS}

To explored whether effect of IncRNA HOTAIR knockdown in LPS-induced ALI, A549 cells were transfected with NC-pcDNA 3.1, HOTAIR-pcDNA 3.1, si-NC and si-HOTAIR for $48 \mathrm{~h}$ and treated with or without LPS for $24 \mathrm{~h}$. As shown as Fig. 2a, the expression of HOTAIR in the HOTAIR-pcDNA 3.1 group was more 20 times higher than other groups without LPS. Compared with the si-HOTAIR groups, the expression of HOTAIR in the si-NC group was more 3 times higher without LPS. Moreover, the expression of HOTAIR in the HOTAIR-pcDNA 3.1 group was also more 20 times higher than other groups and the expression of HOTAIR in the si-NC group was more 5 times higher with LPS (Fig. 2b).

To explore the effect of over-expression and knockdown of HOTAIR on LPS-induced inflammatory response in ALI, A549 cells were transfected with NC-pcDNA 3.1, HOTAIR-pcDNA 3.1, si-NC and si-HOTAIR or $48 \mathrm{~h}$ and treated with LPS for $24 \mathrm{~h}$. As shown as Fig. 3a, the results showed that the expression of IL$1 \beta$, IL- 6 and TNF-a was remarkably increased in the HOTAIR-pcDNA 3.1 group compared with the other groups. ELISA results demonstrated that the expression of IL-1 $1 \beta$, IL- 6 and TNF- $\alpha$ was significantly decreased in the si-HOTAIR group compared with the other groups, (Fig. 3b).

\section{The role of HOTAIR in modulating NF-KB pathway activation}

To investigate over-expression and knockdown of HOTAIR on the activation of NF-kB pathway, A549 cells were transfected with NC-pcDNA 3.1, HOTAIR-pcDNA 3.1, si-NC and si-HOTAIR and treated with LPS. Our result showed that the levels of $p-I_{\kappa} B a / I_{k B a}$ and $p-p 65 / p 65$ were increased in the HOTAIR-pcDNA 3.1 group and decreased in the si-HOTAIR group (Fig. 4).

\section{Knockdown HOTAIR inhibit nuclear translocation of p65 induced by LPS}

The nuclear translocation of p65 NF-kB induced by LPS in A549 cells was then detected by immunofluorescence assay. Our result showed that p 65 NF-kB protein level in the nucleus was 
significantly higher in the HOTAIR-pcDNA 3.1 group than the other groups. P65 NF-KB protein levels in the nucleus was the lowest in si-HOTAIR groups compared with the other groups (Fig. 5).

\section{Discussion}

Our study revealed that the expression level of cytokines (IL-1 $\beta$, IL-6, TNF-a) and inflammatory gene (HOTAIR) were increased in LPS-induced ALI model. Knockdown of HOTAIR can inhibit the secretion of IL$1 \beta$, IL-6, and TNF-a via inhibiting p65 transfer from cytoplasm to nucleus.

More and more evidence showed that non-coding RNA (ncRNA) is an important part of various cellular and physiological signaling processes including ALI [7][15][16][17]. HOTAIR is an antisense transcript that play a role in suppression [18][19][20]. HOTAIR was reported to participate in various cancer and other diseases like acute myocardial infarction, and also involved in immune and inflammatory responses [20] [21][22][23][24]. Monira et al suggests that HOTAIR was a key factor in NF-KB activation of macrophages, regulated the activation of NF-KB and its target genes expression of IL- 6 and iNOS by promoting the degradation of IKBa [24]. Human immune response is complex biochemical processes,modulated by various proteins and lipids[25]. Among them, Toll-like receptors play an important role in the innate response to bacterial infections and to the immune response to pathogens [26][27]. TLR4 activation triggers the activation of NF-KB, inducing increased expression of downstream signaling cascades including cytokines, chemokines, and pro-inflammatory genes. A variety of extracellular stimulation signals including Inflammatory factors, LPS, mitogens of T cells and B cells, viral double-stranded RNA and various physical and chemical pressures was able to trigger the activation of NF-KB signaling pathway[28][29][30][31]. LPS triggers the body's inflammatory response by activating the classic NF-KB pathway, thereby promoting the expression of cytokines such as IL-1, IL- 6 and TNF-a and these cytokines can also induce NF-KB activation[32]. In this study, we explored the potential role of HOTAIR in the regulation of cytokines and immune response in A549 cells. Our results showed that LPS-induced ALI increased the expression of various cytokines including IL-1 $\beta$, IL- 6 and TNF- $a$, and the expression of inflammatory gene HOTAIR was also up-regulated. This result suggests that there may be a correlation between the expression of HOTAIR and cytokines in LPS-induced ALI.

We knockdown HOTAIR and detecting the expression of IкB protein and NF-KB member $\mathrm{p} 65$ for further exploring whether HOTAIR increase the expression of related inflammatory factors by activating NF-KB in LPS-induced ALI. Our results suggest that knockdown HOTAIR significantly decreased the level of $\mathrm{p}-\mathrm{IKBa}$ and p-p65. As we know that in the classical pathway of NF-KB, the activation of IKK kinase complex can phosphorylates $\mathrm{IKBa}$, and further triggering its polyubiquitination and proteasome degradation, leading to the release and activation of NF-KB dimer, which accompanied by the activation of p65[32][33][34]. Ozes et al found that HOTAIR regulated NF-KB by decreased IKBa transcription during DNA damage response) in ovarian cancer[35]. Park et al have reported that the activity of the NF-kB pathway can be influenced by the expression of IncRNA HOTAIR[36]. Therefore, the decreased level of p-IKBa and p-p65 indicates the effective inhibition of NF-KB pathway by HOTAIR knockdown in our study. In addition, our immunofluorescence results indicate that the nuclear translocation of p65 in LPS-induced ALI was 
inhibited after HOTAIR knockdown. Studies have shown that the inhibition of p65 nuclear translocation is also observed after LPS stimulation of macrophages [24]. Thus, our result indicates that HOTAIR may be involved in the degradation of IKBa and activation of NF-KB in LPS-induced ALI.

\section{Conclusion}

In summary, based on our biochemical studies in A549 cells, it is proved that HOTAIR triggered NF-KB activation and promote pro-inflammatory response in LPS-induced ALI, which may provide a perspective for further understanding the pathogenic mechanism of ALI.

\section{Abbreviations}


Non-coding RNA

HOX transcript antisense RNA

Acute lung injury

Lipopolysaccharides

Activation of nuclear factor kappa-B

Inhibitor of NF-KB

IKB kinase

Tumornecrosis factor

Interleukin

Inducible nitric oxide synthases

Toll-like receptor 4

Nuclear paraspeckle assembly transcript 1

Metastasis-associated lung adenocarcinoma transcript 1

Taurine Up-regulated Gene 1

GRB2 associated binding protein 1

High Mobility Group Protein B1/Receptor for Advanced Glycation Endproducts

4',6-Diamidino-2-Phenylindole, Dihydrochloride

Enzyme-linked immunosorbent assay

quantitative Real Time Polymerase Chain Reaction

Paraformaldehyde

Polyvinylidene fluoride

PBS with $0.1 \%$ Tween-20

Horseradish eroxidase

Glyceraldehyde-3-phosphate dehydrogenase

One-way analysis of variance

Statistical package for the social sciences

Standard deviation
ncRNA

HOTAIR

ALI

LPS

NF-KB

IKB

IKK

TNF

IL

iNOS

TLR4

NEAT1

MALAT1

TUG1

GAB1

HMGB1/RAGE

DAPI

ELISA

qRT-PCR

PFA

PVDF

PBST

HRP

GAPDH

ANOVA

SPSS

SD 


\section{Declarations}

\section{Ethics approval and consent to participate:}

Not applicable.

\section{Consent for publication:}

Not applicable.

\section{Availability of data and material:}

The datasets supporting the conclusions of this article are included within the article.

\section{Competing interests:}

None.

\section{Funding:}

This work was supported by Guangzhou Science and Technology Plan Project (Grant 202002030036; received by SQW). The funders had no role in study design, data collection and analysis, decision to publish, or preparation of the manuscript.

\section{Authors`contributions:}

$\mathrm{XMH}, \mathrm{ZXM}$ and SQW contributed to the literature search and study design, ZXM contributed to the analysis of data, XMH contributed to the manuscript preparation, $\mathrm{HH}, \mathrm{KWW}, \mathrm{NNX}$, WHG and WNT collecting data, YJL and SQW contributed to the review of manuscript. All authors read and approved the final manuscript.

\section{Acknowledgement}

We thank XiaoLiang Liang for assistance with valuable discussion, meanwhile, we thank for the editors and reviewers for their valuable comments on the paper.

\section{References}

1. Rubenfeld GD, Caldwell E, Peabody E, Weaver J, Martin DP, Neff M, et al. Incidence and outcomes of acute lung injury. N Engl J Med. 2005;353:1685-93.

2. Zhang B, Liu ZY, Li YY, Luo Y, Liu ML, Dong HY, et al. Antiinflammatory effects of matrine in LPSinduced acute lung injury in mice. Eur J Pharm Sci. 2011;44:573-9.

3. Ghosh S, Hayden MS. New regulators of NF-KB in inflammation. Nat Rev Immunol. 2008;8:837-48. 
4. Welbourn CRB, Young Y. Endotoxin, septic shock and acute lung injury: Neutrophils, macrophages and inflammatory mediators. Br J Surg. 1992;79:998-1003.

5. Bhatia M, Moochhala S. Role of inflammatory mediators in the pathophysiology of acute respiratory distress syndrome. J Pathol. 2004;202:145-56.

6. Flynn RA, Chang HY. Long noncoding RNAs in cell-fate programming and reprogramming. Cell Stem Cell. 2014;14:752-61.

7. Zhou H, Wang X, Zhang B. Depression of IncRNA NEAT1 Antagonizes LPS-Evoked Acute Injury and Inflammatory Response in Alveolar Epithelial Cells via HMGB1-RAGE Signaling. Mediators Inflamm. 2020;2020:8019467.

8. Hu H, Wu J, Yu X, Zhou J, Yu H, Ma L. Long non-coding RNA MALAT1 enhances the apoptosis of cardiomyocytes through autophagy inhibition by regulating TSC2-mTOR signaling. Biol Res. 2019;52:130-6.

9. Zhu W, Men X. Negative feedback of NF-KB signaling by long noncoding RNA MALAT1 controls lipopolysaccharide-induced inflammation injury in human lung fibroblasts WI-38. J Cell Biochem. 2020;121:1945-52.

10. Liang WJ, Zeng XY, Jiang SL, Tan HY, Yan MY, Yang HZ. Long non-coding RNA MALAT1 sponges miR-149 to promote inflammatory responses of LPS-induced acute lung injury by targeting MyD88. Cell Biol Int. 2020;44:317-26.

11. Qiu N, Xu X, He Y. LncRNA TUG1 alleviates sepsis-induced acute lung injury by targeting miR-34b5p/GAB1. BMC Pulm Med. 2020;20:49.

12. Tang Q, Hann SS. HOTAIR: An oncogenic long non-coding RNA in human cancer. Cell Physiol Biochem. 2018;47:893-913.

13. Loewen G, Jayawickramarajah J, Zhuo Y, Shan B. Functions of IncRNA HOTAIR in lung cancer. Journal of Hematology Oncology. 2014;7:90.

14. Wei S, Wang K, Huang X, Tang W, Zhao Z, Zhao Z. Knockdown of the IncRNA MALA T1 alleviates lipopolysaccharide-induced A549 cell injury by targeting the miR-17-5p/FOXA1 axis. Mol Med Rep. 2019;20:2021-9. doi:10.3892/mmr.2019.10392.

15. Su Q, Liu Y, Lv XW, Dai RX, Yang XH, Kong BH. LncRNA TUG1 mediates ischemic myocardial injury by targeting miR-132-3p/HDAC3 axis. Am J Physiol Heart Circ Physiol. 2020;318:H332-44.

16. Guttman M, Amit I, Garber M, French C, Lin MF, Feldser D, et al. Chromatin signature reveals over a thousand highly conserved large non-coding RNAs in mammals. Nature. 2009;458:223-7.

17. Zhang J, Mao F, Zhao G, Wang H, Yan X, Zhang Q. Long non-coding RNA SNHG16 promotes lipopolysaccharides-induced acute pneumonia in A549 cells via targeting miR-370-3p/IGF2 axis. Int Immunopharmacol. 2020;78:106065.

18. Rinn JL, Kertesz M, Wang JK, Squazzo SL, Xu X, Brugmann SA, et al. Functional Demarcation of Active and Silent Chromatin Domains in Human HOX Loci by Noncoding RNAs. Cell. 2007;129:1311-23. 
19. Elsayed ET, Salem PE, Darwish AM, Fayed HM. Plasma long non-coding RNA HOTAIR as a potential biomarker for gastric cancer. Int J Biol Markers. 2018;33:528-33.

20. Xiao Z, Qu Z, Chen Z, Fang Z, Zhou K, Huang Z, et al. LncRNA HOTAIR is a Prognostic Biomarker for the Proliferation and Chemoresistance of Colorectal Cancer via MiR-203a-3p-Mediated Wnt/ßCatenin Signaling Pathway. Cell Physiol Biochem. 2018;46:1275-85.

21. Cai B, Song XQ, Cai JP, Zhang S. HOTAIR: A cancer-related long non-coding RNA. Neoplasma. 2014;61:379-91.

22. Li L, Zhang M, Chen W, Wang R, Ye Z, Wang Y, et al. LncRNA-HOTAIR inhibition aggravates oxidative stress-induced H9c2 cells injury through suppression of MMP2 by miR-125. Acta Biochim Biophys Sin (Shanghai). 2018;50:996-1006.

23. Zhang $\mathrm{H}$, ju, Wei Q feng, Wang $\mathrm{S}$ jun, Zhang $\mathrm{H}$, Zhang $\mathrm{X}$, ying, Geng Q, et al. LncRNA HOTAIR alleviates rheumatoid arthritis by targeting miR-138 and inactivating NF-KB pathway. Int Immunopharmacol. 2017;50:283-90.

24. Obaid M, Udden SMN, Deb P, Shihabeddin N, Zaki MH, Mandal SS. LncRNA HOTAIR regulates lipopolysaccharide-induced cytokine expression and inflammatory response in macrophages. Sci Rep. 2018;8:15670.

25. Chaplin DD. Overview of the immune response. J Allergy Clin Immunol. 2010;125 2 SUPPL. 2.

26. Vasselon T, Detmers PA. Toll receptors: A central element in innate immune responses. Infect Immun. 2002;70:1033-41.

27. Baral P, Batra S, Zemans RL, Downey GP, Jeyaseelan S. Divergent functions of toll-like receptors during bacterial lung infections. Am J Respir Crit Care Med. 2014;190:722-32.

28. Lu YC, Yeh WC, Ohashi PS. LPS/TLR4 signal transduction pathway. Cytokine. 2008;42:145-51.

29. Wang YY, Sun SP, Zhu HS, Jiao XQ, Zhong K, Guo YJ, et al. GABA regulates the proliferation and apoptosis of MAC-T cells through the LPS-induced TLR4 signaling pathway. Res Vet Sci. 2018;118:395-402.

30. Kawai T, Takeuchi O, Fujita T, Inoue J, Mühlradt PF, Sato S, et al. Lipopolysaccharide Stimulates the MyD88-Independent Pathway and Results in Activation of IFN-Regulatory Factor 3 and the Expression of a Subset of Lipopolysaccharide-Inducible Genes. J Immunol. 2001;167:5887-94.

31. Kuzmich NN, Sivak KV, Chubarev VN, Porozov YB, Savateeva-Lyubimova TN, Peri F. TLR4 signaling pathway modulators as potential therapeutics in inflammation and sepsis. Vaccines. 2017;5:34.

32. Li Q, Verma IM. NF-kB regulation in the immune system. Nat Rev Immunol. 2002;2:725-34.

33. Jeong SJ, Pise-Masison CA, Radonovich MF, Hyeon UP, Brady JN. A novel NF-KB pathway involving IKKßand p65/RelA Ser-536 phosphorylation results in p53 inhibition in the absence of NF-KB transcriptional activity. J Biol Chem. 2005;280:10326-32.

34. Hochrainer K, Racchumi G, Anrather J. Site-specific phosphorylation of the p65 protein subunit mediates selective gene expression by differential NF-KB and RNA polymerase II promoter recruitment. J Biol Chem. 2013;288:285-93. 
35. Özeş AR, Miller DF, Özeş ON, Fang F, Liu Y, Matei D, et al. NF-kB-HOTAIR axis links DNA damage response, chemoresistance and cellular senescence in ovarian cancer. Oncogene. 2016;35:5350-61.

36. Park G, Bin, Chung YH, Jeong JY, Kim D. A p1108-specific inhibitor combined with bortezomib blocks drug resistance properties of EBV-related B cell origin cancer cells via regulation of NF-KB. Int J Oncol. 2017;50:1711-20.

\section{Figures}

a

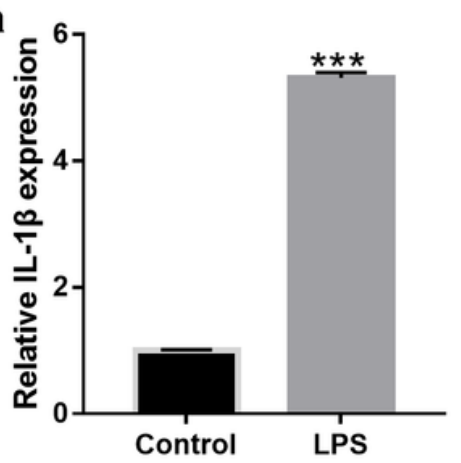

b

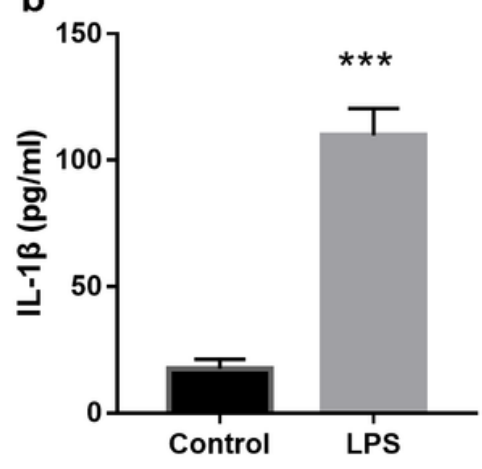

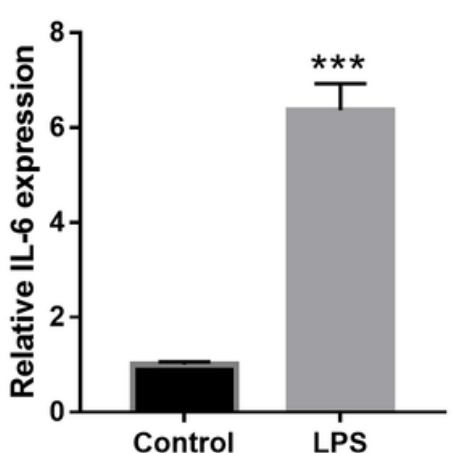

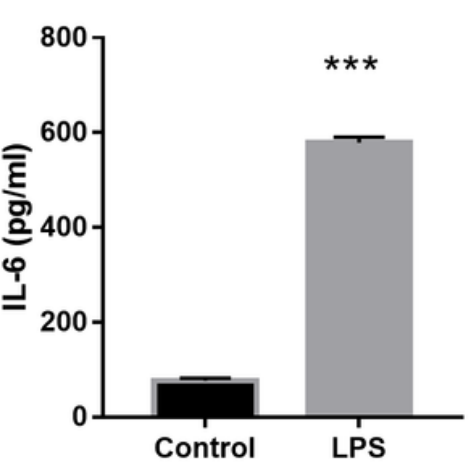

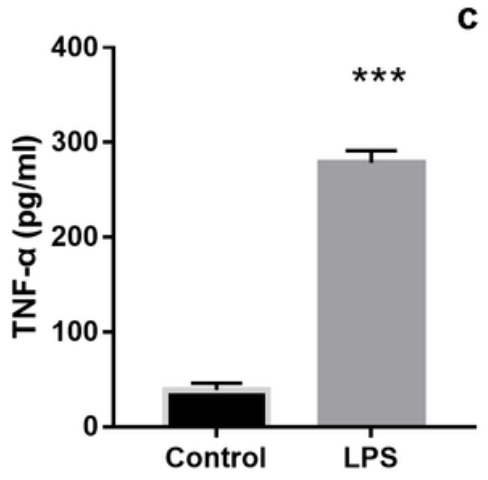

C
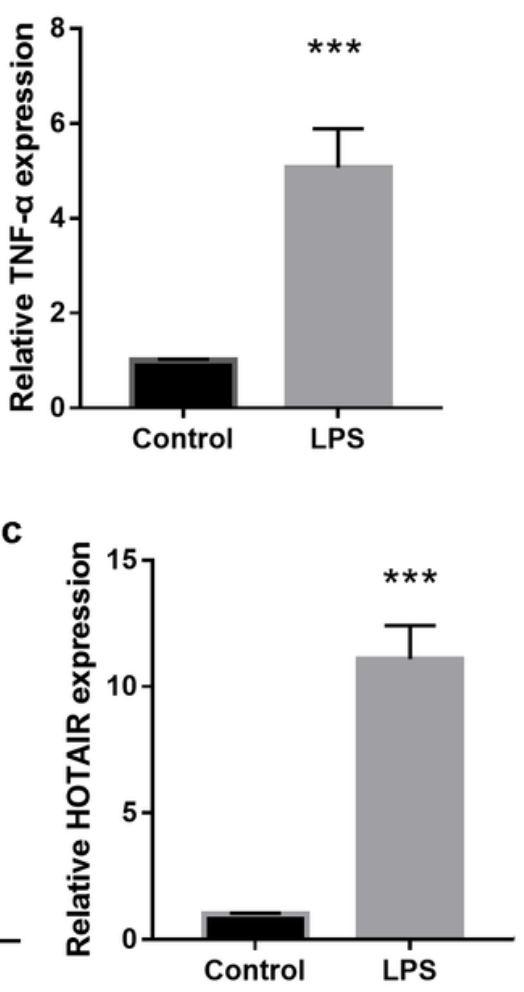

Figure 1

LPS promotes IL-1 $\beta$, IL-6, TNF- $\alpha$ and IncRNA HOTAIR expression. (a) Increased expression of IL-1 $\beta$, IL-6 and TNF- $\alpha$ in LPS-induced ALI detected by qRT-PCR. (b) Increased expression of IL-1 $\beta$, IL- 6 and TNF- $\alpha$ in LPS-induced ALI detected by ELISA. (c) Increased expression of IncRNA HOTAIR in LPS-induced ALI detected by qRT-PCR. Each experiment was repeated three times. ${ }^{*} * \mathrm{P}<0.001$. 
a

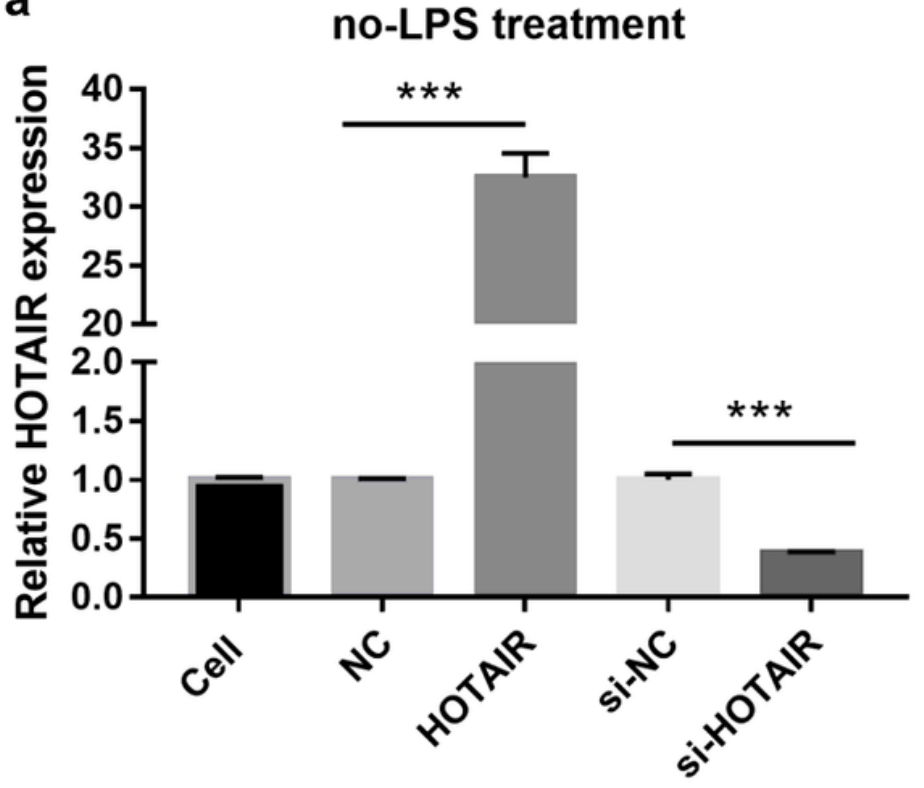

b

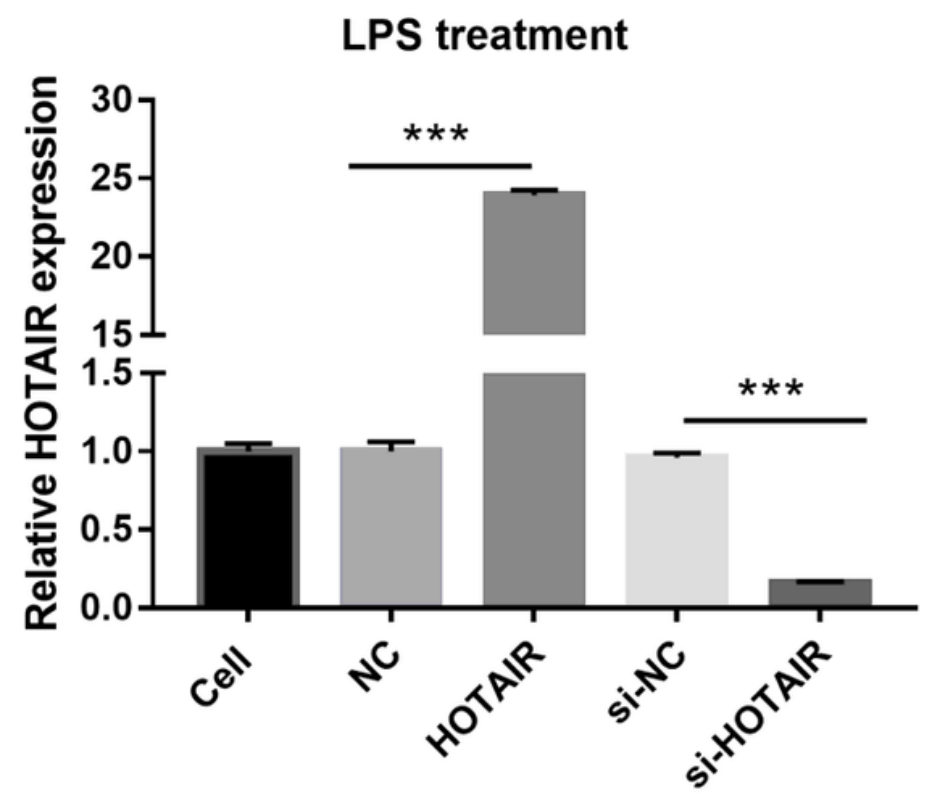

Figure 2

Overexpression and knockdown of IncRNA HOTAIR with/without LPS stimulation. (a) RT-qPCR analysis of HOTAIR expression in A549 cells after transfected with NC-pcDNA 3.1, HOTAIR-pcDNA 3.1, si-NC and si-HOTAIR for $48 \mathrm{~h}$ and treated without LPS for 24h. (b) RT-qPCR analysis of HOTAIR expression in A549 cells after transfected with NC-pcDNA 3.1, HOTAIR-pcDNA 3.1, si-NC and si-HOTAIR for 48h and treated with LPS for $24 \mathrm{~h}$. ${ }^{* * *} \mathrm{P}<0.001$.

a

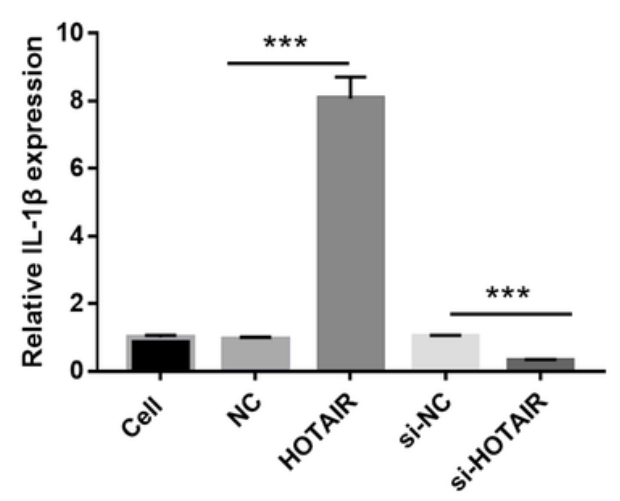

b

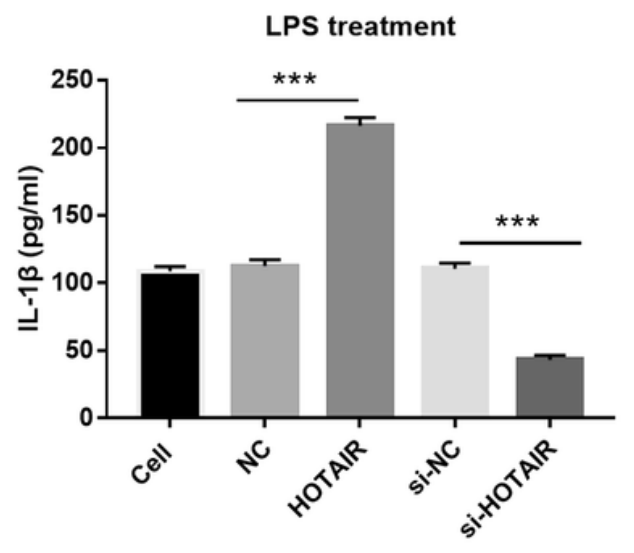

LPS treatment
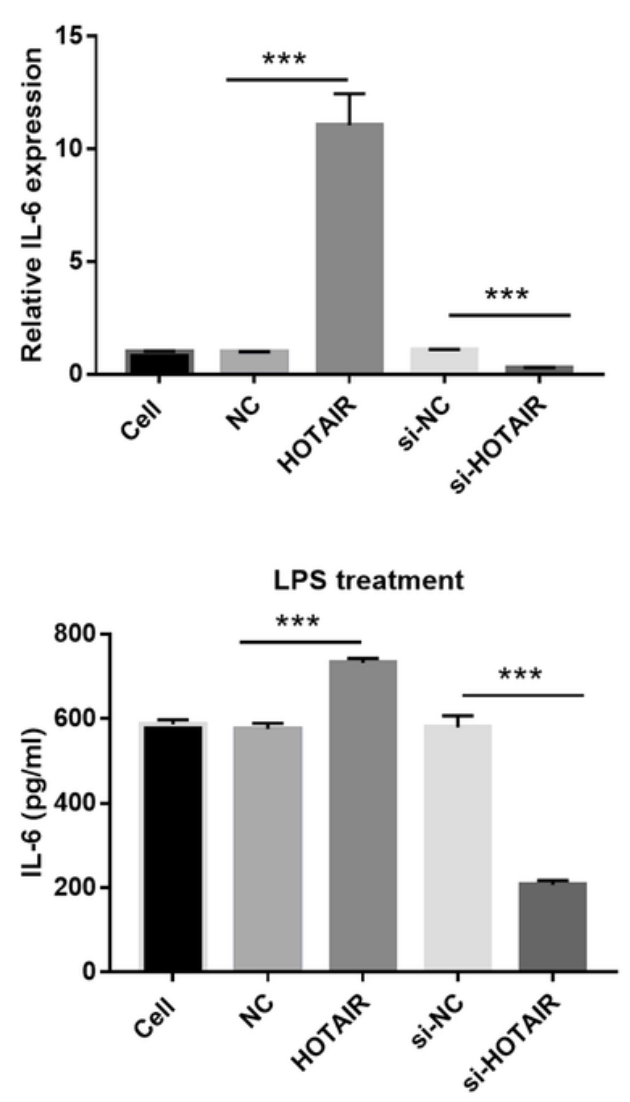

LPS treatment

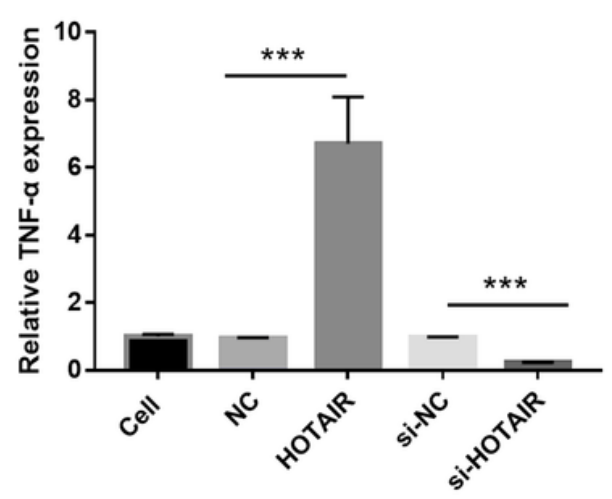

LPS treatment

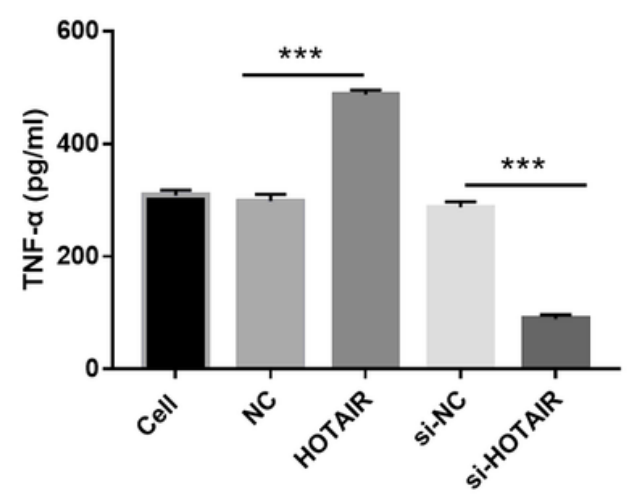




\section{Figure 3}

Overexpression and knockdown of HOTAIR on LPS-induced inflammatory response. (a) RT-qPCR analysis of HOTAIR expression in A549 cells after transfected with NC-pcDNA 3.1, HOTAIR-pcDNA 3.1, si-NC and si-HOTAIR for $48 \mathrm{~h}$ and treated with LPS for 24h. (b) ELISA assay of IL-1 $\beta$, IL-6 and TNF-a level in the culture supernatant in A549 cells, which were transfected with NC-pcDNA 3.1, HOTAIR-pcDNA 3.1, si-NC and si-HOTAIR for $48 \mathrm{~h}$ and treated with LPS for $24 \mathrm{~h}$. *** $\mathrm{P}<0.001$. GADPH
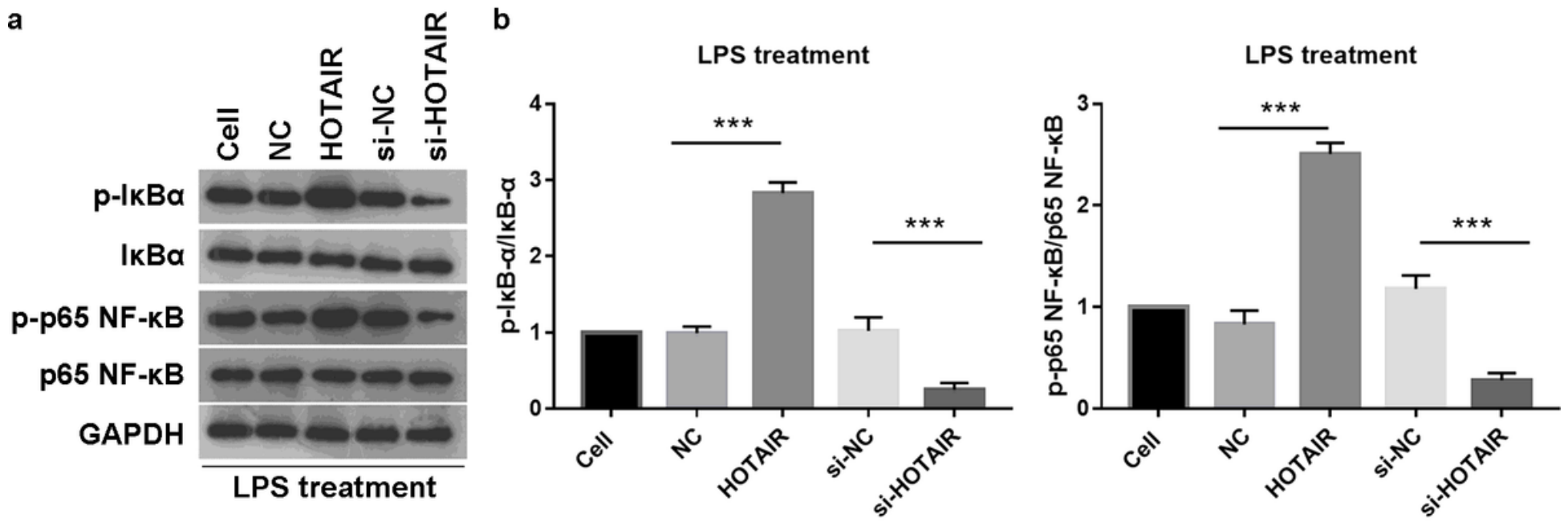

Figure 4

Overexpression and knockdown of HOTAIR on NF-kB pathway by treated with LPS. A549 cells after transfected with NC-pcDNA 3.1, HOTAIR-pcDNA 3.1, si-NC and si-HOTAIR for 48h and treated with LPS for 24h. (a) Increased levels of $p$-IкBa, IкBa, p-p65 NF-kB and p65 NF-kB in HOTAIR-pcDNA 3.1 group and significantly decreased level in si-HOTAIR group by western blot. (b) The relative expression levels of $p$ $\mathrm{IkBa} / \mathrm{lkBa}$ and p-p65 NF-kB and p-p65 NF-kB/NF-kB were increased in HOTAIR-pcDNA 3.1 group and significantly decreased level in si-HOTAIR group. ${ }^{\star \star *} \mathrm{P}<0.001$. 


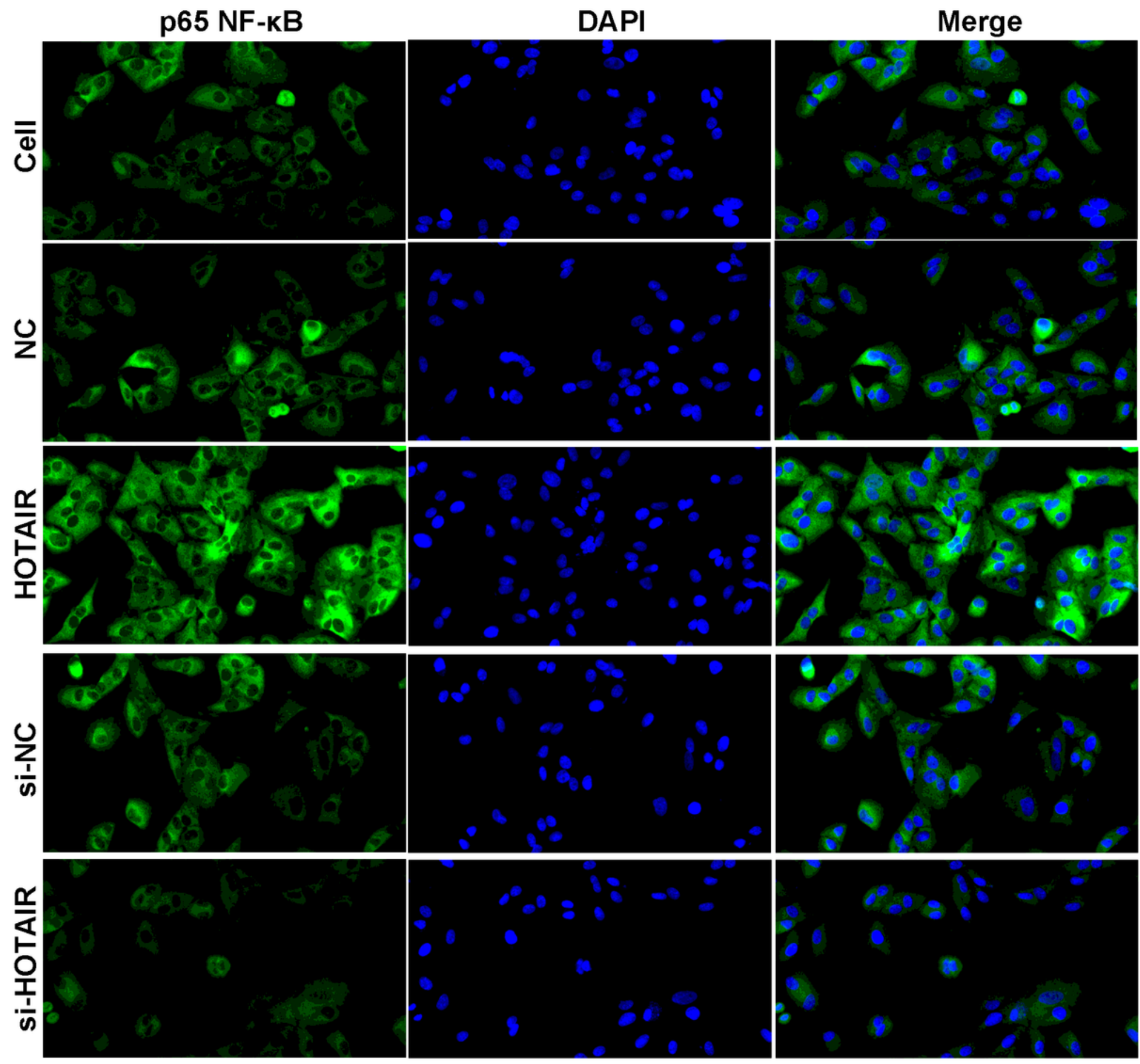

Figure 5

Knockdown of HOTAIR inhibited LPS-induced translocation of p65 NF-kB protein from cytoplasm to nucleus. A549 cells after transfected with NC-pcDNA 3.1, HOTAIR-pcDNA 3.1, si-NC and si-HOTAIR for 48h and treated with LPS for $24 \mathrm{~h}$. P65 NF-KB protein level in the nucleus was significantly higher in the HOTAIR-pcDNA 3.1 group than the other groups. Images were taken by fluorescence microscope (Magnification, 400x).

\section{Supplementary Files}


This is a list of supplementary files associated with this preprint. Click to download.

- GelBMC.tif 